\title{
A propósito de lo hipermaterial ${ }^{*}$
}

\author{
Entrevista de Vincent Bontems a Bernard Stieglep \\ Traducción del francés al español de Luis Alfonso Paláu-Castaño \\ Universidad Nacional de Colombia, Medellín, Colombia \\ lapalau@gmail.com
}

Vincent Bontems: Entre los diversos motivos que usted desarrolla con miras a la aprensión y a la transformación del mundo contemporáneo, su filosofía hace del desarrollo tecnológico industrial uno de sus principales temas de reflexión, y lo caracteriza como habiendo alcanzado el estadio hiperindustrial. Según usted ¿cuáles son las tentaciones que se han hecho posibles por lo que se llama "lo inmaterial"?

Bernard Stiegler: La tentación es una cuestión esencial y permanente en la existencia del ser no-inhumano. Simondon $(2007,2009)$ lo ha establecido perfectamente. La tentación puede ser un tema religioso, pero marca mucho más profundamente como su ley toda la organización psíquica y social en tanto que proceso dinámico en donde se enfrentan tendencias. Es algo indudable que haya en nuestros días tentaciones de todo género, a la vez las insertas en un fondo muy arcaico de tendencias primarias y las que son por completo específicas de nuestra época y de las posibilidades insignes que aquí abre su devenir tecnológico; que tales tentaciones sean un problema, es decir, que puedan conducir a procesos regresivos y que esas formas regresivas sean ellas mismas completamente específicas de nuestra época.

Se trataría, sin embargo, de saber si las tentaciones de lo inmaterial son claramente una cuestión, y cómo es necesario entender esa cuestión, ya sea buena o mala. ¿Cuál es la intención que se mantiene tras esta pregunta? ¿Hacia qué tiende esta pregunta? Comencemos por tratar de descubrir esta intención. Quizás me equivoque, pero me parece que esto sugiere la idea de que, con estas tecnologías (que no diría que son, por mi parte, tecnologías de lo "inmaterial", sino tecnologías de la información, de la comunicación, tecnologías cognitivas

Cómo citar: Bontems, V. (2020). A propósito de lo hipermaterial. Ciencias Sociales y Educación, 9(18), 261-274. https://doi.org/10.22395/csye.v9n18a12

Traducción realizada por Luis Alfonso Palau-Castaño del texto de Bontems, (2008).

Recibido: 10 de abril de 2020.

Aprobado: 21 de agosto de 2020. 
y culturales), tenemos que vérnoslas con lo que Gilles Deleuze (2003) llamaba las "tecnologías de control". Si puede haber aquí una cuestión original de la tentación con tales tecnologías, es porque ellas abren posibilidades antagónicas en las cuales el poder está tentado a privilegiar y monopolizar una tendencia, o un haz de tendencias, entre estas posibilidades; tiene la tentación de hacer de ello exclusiva y hegemónicamente las tecnologías de su poder y hablo aquí, en este caso, del poder propio de la época, la de la conversión del capital en financiero. Esta tentación del poder es, sin embargo, un error, pues ella se revela autodestructiva a corto plazo.

Se trata, sin la menor duda - con las tecnologías que yo creo son las de lo hipermaterial mucho más que de lo inmaterial-, de dispositivos de poder absolutamente temibles. Estos dispositivos hipermateriales permiten desarrollar lo que podríamos llamar, para ir rápido, las tecnologías de un psicopoder, las cuales instalan situaciones masivas de servidumbre voluntaria, fascinación y anestesia sin equivalente en la historia. Ahora bien, se puede estar, por lo demás, tentado a generalizar las conexiones entre estas tecnologías cognitivas y culturales y las tecnologías materiales del armamento, de la vigilancia, de los captores, pasando, por supuesto, por todo lo que se pone ya en su sitio en internet como trazabilidad y análisis automático de los comportamientos individuales, etc.. Esta integración generalizada conduce finalmente a un nuevo sistema panóptico en los límites de lo que permite la electrónica, que podríamos calificar de "nanotrónica".

Vincent Bontems: En esta perspectiva, ¿las tecnologías de la información serían eventualmente portadoras de alienación?

Bernard Stiegler: Absolutamente evidente. Incluso esto ha comenzado ya desde hace tiempos; era una de las conclusiones más importantes del reporte de Simon Nora y de Alain Minc a Valéry Giscard d'Estaing hace exactamente treinta años, y esto podría llegar extremadamente lejos.

Acabo de hacer la recensión de un libro de Philippe Lemoine $(2007)^{1}$, donde él evoca esta posibilidad que ofrece en teoría el IPV6 ${ }^{2}$ de codificar 2128 direcciones electrónicas, lo que es superior al número de átomos de la superficie de la tierra; se podrían codificar todos los objetos que existen a nivel atómico. Esta situación borgesiana evidentemente produce miedo por las posibilidades y, por tanto, por las fenomenales tentaciones de control. Hablar así, ciertamente, es algo demasiado superficial, y vamos a tratar de profundizar la cuestión. Pero yo

Philippe Lemoine es el PDG (presidente director general) de Laser.

2 IPV6: Internet Protocol Version 6 (para la dirección sobre internet, V6 es la futura versión). 
creo que hay - cualesquiera que sean las tentaciones posibles - una realidad nueva que tiene que ver con el desarrollo del control comportamental individual y colectivo a través de estas tecnologías. Y esta realidad, que se juega ante todo en el dominio de los mass-media, ejerce sus efectos en el comportamiento de los consumidores.

Vincent Bontems: En este sentido, el término "tentación" es afortunado pues, para establecerse, este control debe ejercerse a través de los "espejismos", es decir, de las cosas que buscan reemplazar la presa por su sombra. ¿La "tentación" no será para cada uno de nosotros sufrir la fascinación y adherirse —incluso con nuestro cuerpo que se defiende sin inintención-a la aparición de este sistema de regulación, que es coercitivo seguramente, pero de una manera paradójica, puesto que pasa por la responsabilidad de cada uno de desresponsabilizarse?

Bernard Stiegler: En efecto, y este es el regreso masivo de lo que Kant llamaba la minoría de edad. Se debe también entender "tentación" en este otro sentido, es decir, desde el punto de vista del que padece. Pues, para que yo esté tentado a manipular a otro es necesario que él esté tentado por lo que yo le propongo. Esto solo es posible porque en cada uno de nosotros hay una tendencia al abandono; la tentación de la renuncia y, aún más profundamente, la tentación de dejarse llevar por la pulsión. Volveré sobre el asunto.

$\mathrm{El}$ otro aspecto interesante de su pregunta es el de la irresponsabilidad. Vivimos en una sociedad que reposa sobre la irresponsabilización tendencial del consumidor; el consumidor debe ser también, en lo posible, completamente irresponsable, sobre todo si debe ser un consumidor de servicios, aunque también pase con el consumidor de objetos. El consumidor ideal es el que consume lo más posible. Esto no es verdad en todos los dominios; aún hay productos, medicamentos, en los cuales se establecen límites para lo que se prescribe. Pero, por regla general, la tendencia es empujar el consumo a su máximo. Por consiguiente, hay un fenómeno de desrresponsabilización, o de irresponsabilización de los consumidores, que es una tentación extremadamente peligrosa. Desafortunadamente, ella ha sido ya ampliamente satisfecha. Se perciben sus consecuencias desastrosas a través de la problemática llamada"desarrollo durable", que está confrontado con el hecho de que el sistema alcanza los límites de funcionamiento del consumo de los recursos naturales y que es generador de una toxicidad planetaria - tanto física como mental— que se vuelve insostenible. Es un primer punto.

Pasemos a lo "inmaterial". Yo no creo en lo inmaterial, eso no existe. Es una palabra fácil que emplean gentes que a veces están en el primer rango, como 
André Gorz (2003), y que designa, de hecho, estados de la materia evanescentes, pero que siguen siendo estados de la materia. No existe nada que no sea un estado de la materia. Y para producir esos estados evanescentes, se requiere mucho material, muchos aparatos, aunque nosotros estemos, más bien, en una economía y una época de la "hipermateria" tanto como de lo "hipermaterial".

Llamo hipermateria a un complejo de energía y de información en el que ya no es posible distinguir la materia de su forma; es lo que aparece con la mecánica cuántica y lo que necesita el rebasamiento de lo que Simondon (2007) llama el esquema hilemórfico, es decir, la manera de pensar según una pareja de conceptos, la forma (morphé) y la materia (hylé), que consiste en pensarlas oponiéndolas. Y llamo hipermaterial a un proceso en el que la información —que se presenta como una forma - es en realidad un tren de estados de materia producido por materiales, aparatos o dispositivos tecnológicos, donde la separación de la materia y de la forma está aquí totalmente desprovista de sentido.

En el plano de la vida cotidiana no se asiste de ninguna manera a una desmaterialización, sino, por el contrario, a una hipermaterialización; todo se ha transformado en información, es decir, en estados de materia por la intermediación de materiales y de aparatos, lo que hace a todo esto controlable a nivel del nanómetro y del nanosegundo. Este proceso conduce a una extensión siempre más considerable de los estados accesibles de la materia, portadora de forma, que de ahora en adelante tiene la capacidad de trabajar en lo infinitamente pequeño y en lo infinitamente breve. De repente, la materia se vuelve invisible. El problema no es, pues, la inmaterialidad, sino la invisibilidad de la materia (lo que han comprendido muy bien Xavier Guchet y Sacha Loève3, cuando hablan de la biometría o de las microtecnologías). Lo que conecta con su pregunta sobre el espejismo.

Vincent Bontems: ¿No hay, en efecto, una conexión notable entre esos estados sutiles, imperceptibles y, por tanto, invasivos de la materia, además de toda una industria del "hacer visible" que genera la adhesión a través de emociones de orden estético gracias a imágenes?

Bernard Stiegler: Las tecnologías de representación son también tecnologías de manipulación. Ya escribí sobre estas cuestiones (Stiegler, 2006); entonces prefiero ahondar la cuestión de la llamada "inmaterialidad", que yo describo (al contrario de la doxa dominante) como esta hipermaterialidad cuya expan-

Maestro de conferencia en filosofía en la universidad Paris-1 (Sorbona), Xavier Guchet anima con Sacha Loève, estudiante de doctorado, el seminario "Nanotecnologías" en el Instituto de investigación y de innovación del Centro Georges Pompidou, creado y dirigido por Bernard Stiegler. 
sión económica, bajo el control del capital financiero y a su exclusivo servicio, vivimos en nuestros días. Corresponde a un nuevo estadio de la gramatización (Stiegler, 2006, pp. 15, 21-25), es decir, del proceso de discretización que abre, pues, la posibilidad de una indización de la materia misma. En este sentido, la hipermateria es también una materia portadora de sus propios metadatos, como se dice en las tecnologías cognitivas. La naturaleza muestra ser aquí —o más bien devenir- un libro, como lo decía Tommaso Campanella. El bibliómeno y la fenomenotécnica pensadas por Bachelard toman acá un nuevo giro.

Más inmediatamente, vivimos una época en que los procesos motores y mentales de los sistemas nerviosos centrales pueden ser replicados, copiados, grabados, transformados, exteriorizados y, por tanto, materializados bajo otras formas. Por ejemplo, se puede pasar de algo que está materializado por medio de un cierto estado de mi cerebro, una actividad de mi laringe, mi caja torácica —que se llama mi voz- la que alcanza sus oídos bajo forma de trenes de ondas (que se podrían visualizar con un osciloscopio), al registro numérico (una serie de ceros y de unos) en este dictáfono - o lo que haga sus veces-, que es en este caso una llave USB, es decir, un soporte de silicio; lo inmaterial no existe, nunca ha existido, jamás existirá.

En desquite, la información pretendidamente "desmaterializada" es transcodificada por discretización, luego transmitida, lo que hace que sea pensada analógicamente como siendo inmaterial con respecto a su soporte de origen. Pero en realidad ella está siempre ya informada por otra forma material. Esto se ha hecho posible por la "materia inorgánica organizada" (Stiegler, 2002), por la organización técnica de la materia que permite, entre otras cosas, producir los objetos de uso, algo que no se sabía pensar correctamente antes de Gilbert Simondon. La materia inorgánica organizada hace aparecer un nuevo régimen de individuación, entre el de los seres inertes y minerales, y el de los seres orgánicos y vivientes; este tercer reino, el inorgánico organizado, va del sílex tallado hasta este dictáfono USB y, más allá, hasta el IPV6.

La gramatización, en sentido estricto (como sistema discreto), apareció a partir del paleolítico superior con la posibilidad de codificar algo de manera distinta al código donde ya estaba codificada, en particular los procesos mentales. Pensamientos o emociones son transmitidos bajo la forma de una vaca negra en la gruta de Lascaux, tatuajes en un cuerpo del brujo aborigen o también por las famosas conchitas llamadas cauris y de las que dice Jack Goody (1977) que son a la vez medio de cambio y de cálculo entre los africanos del norte de Ghana. Los primeros sistemas de engramas de listas finitas de elementos discretos se desarrollan luego en el neolítico. Pero Platón en el Fedro, va a "olvidar" este proceso echando una prohibición sobre la cuestión que considera 
como sofística, devaluando la hypomnesis con respecto a la anamnesis, haciendo de la reminiscencia pura de toda mnemotécnica el corazón de su dialéctica. Habrá necesidad de esperar dos mil quinientos años, y a Edmund Husserl (2002), para que esta apuesta vuelva a aparecer en la superficie, incluso si Leibniz, Condorcet y algunos otros habían reactivado parcialmente el problema.

En el siglo XVIII, este movimiento se amplía con una gramatización de un nuevo tipo que va a tomar una enorme importancia: la del gesto humano. Adam Smith será el primero en observar precisamente el funcionamiento de una máquina que reproduce un saber obrero. Su análisis será retomado por Karl Marx. Son etapas fundamentales que no tengo aquí el tiempo de analizar en detalle, pero que son esenciales para comprender la emergencia de nuevos tipos de engranajes de la memoria bajo formas perceptivas, auditivas y visuales. Con la era de la reproducibilidad mecanizada — según la expresión de Walter Benjamin (1982), se alcanza un nuevo estadio de la gramatización: el desarrollo de la fotografía, la fonografía, la telefonía y finalmente el cinematógrafo, antes de la radio y la televisión. Este estadio posee una característica inédita con respecto a los que le han precedido: es capaz de reproducir de manera absolutamente exacta el tiempo de los objetos que duplica. De esa manera, está en capacidad de captar la atención de los que contemplan esas reproducciones. Sobre estos señuelos fantásticos he tratado en mi teoría de los "objetos temporales industriales" (Stiegler, 2004a, 2004b) y han tomado una importancia formidable. En el siglo XVIII, un campesino de nuestras provincias solo escuchaba música cuando iba al oficio religioso o cuando participaba de las fiestas del pueblo. En nuestros días, huimos de la música y no sabemos cómo escapar de ella. Se dice ahora que esta constante emisión de sonidos es "invasiva"; nos rodea, nos envuelve y nos invade por todas partes, en el aeropuerto, en el supermercado, donde el peluquero... nos inunda literalmente. Nuestras consciencias son solicitadas permanentemente por los iPads, los teléfonos celulares y muy pronto por la difusión de la televisión en los teléfonos móviles. Hemos logrado una captación de los individuos próxima de las diez horas diarias. Tal es la época de lo que analizo como un psicopoder (Stiegler, 2008).

La gran tentación es querer someter la atención a una captación integral, movilizar todo el "tiempo de cerebro disponible", lo que constituye una destrucción de la atención. Pues la atención es algo que se forma lentamente, a través de un sistema de cuidado complejo, que va de los primeros gestos que la madre consagra al niño de pecho hasta las formas más elaboradas de la sublimación, pasando por todo lo que constituye el superyó. Puedo captar la atención de un animal y crear reflejos condicionados que se parecen a esperas, como Pavlov con su perro; pero esas no son esperas, son comportamientos reflejos y automáticos, es decir, todo lo contrario de una espera, la cual supone precisamente 
una atención. La atención no es simplemente la concentración psicológica, es también un comportamiento social con respecto al otro que aparece como otro, precisamente, comprendido aquí a través de sus objetos que, en tanto que son sus objetos, están catexizados del espíritu del otro, son ya otro, pudiendo este ser anónimo. Pero el objeto o la cosa se cargan entonces con la presencia de eso que llamamos un mundo y, finalmente, el mundo, en tanto que él debe convertirse siempre, mientras que yo sea un ser no inhumano, en el objeto de mi atención.

En un ser religioso, este mundo debe ser el objeto de la atención porque es el de Dios. Pero yo puedo no ser religioso y, sin embargo, ponerle atención al mundo. Esto quiere decir que estoy dotado de un espíritu que me religa a este mundo como siendo el sentimiento de mi responsabilidad en cuanto al porvenir de este mundo, y de mi capacidad para inscribir este porvenir del mundo en el devenir ciego en el que consiste mecánicamente.

Sin embargo, no es demasiado complicado crear reflejos condicionados, es decir, aniquilar toda responsabilidad. Esto conduce a crear circuitos neuronales en el cerebro del perro. Jugando con la plasticidad sináptica, puedo domar un animal: un perro, un toro, un caballo, una aplysia. Nosotros mismos, los seres no inhumanos, podemos ser domados y domesticados así y volvernos domésticos completamente. Compartimos esta posibilidad con la aplysia y la babosa. Y, sin embargo, no estoy de acuerdo con el punto de vista de Peter Sloterdijk (2000) sobre lo que él describe como una "domesticación del ser". Considero que el ser humano no es de modo durable ni domesticable ni amaestrable; no existe técnica de doma del ser no inhumano (el que se llama más corrientemente el ser humano).

Se puede, en desquite, amaestrar un ser inhumano. Usted y yo somos también seres inhumanos, todos los otros seres humanos también son seres inhumanos susceptibles, por ello, de ser domados. Por esto hablo de ser no inhumano, porque el ser humano puede - a través de su proceso de individuación- escapar a la tentación de devenir inhumano. El gran pensamiento simondoniano de la individuación psíquica y colectiva4 consiste en plantear que esta individuación es un proceso metaestable, y que esta metaestabilidad produce permanentemente oscilaciones que se analizan, precisamente, a través de la situación de la tentación. Lo que hace aparecer el pensamiento de la individuación psicosocial es que él hace razonar, no en términos de cualidad del ser (domesticable o no), sino más bien de polaridades en las cuales se componen tendencias sin cesar. Que haya una tendencia y una tentación a la sumisión es algo que solo es posible en la medida en que hay una contratendencia a la responsabilidad y a la acción. Aislar una de las tendencias para ontologizarla es cometer un sofisma, que en

Título de la principal obra de Simondon (2009). 
realidad es una manera de ponerse al servicio de una de esas tendencias. La parte inhumana del ser no inhumano es lo que, en el proceso de individuación, tiende hacia la desindividuación que constituye, sin embargo, un elemento dinámico de la individuación. Traté de mostrar en otra parte cómo la pulsión, en lo que ella tiene de egoísta, es la que da al deseo su energía, pero porque el deseo es el que invierte el signo de esta energía egoísta a la que transforma en una potencia de socialización. Tenemos, pues, que esta parte pulsional -y canalizable a través de los automatismos tales como los condicionamientos pavlovianos que pueden, en efecto, condicionar la plasticidad sináptica del ser humano-, esta parte (cuando se la plantea como una cualidad aislada, que se impondría como domesticidad fundamental del ser, es decir, que tiende a dominar la individuación y finalmente a destruirla), esta parte impuesta por la domesticación efectiva del ser en particular a través de la captación destructiva de su atención está ineluctablemente destinada a volverse loca. Cuando se educa un lobo, en su vejez él regresa al instinto y te salta a la garganta. Es lo que estamos haciendo en este momento con la parte inhumana que hay en nosotros, con ese lobo que está en el hombre y del que habla Hobbes. Hemos captado la atención del lobo, se lo fascina, se le ha dado una fuerza enorme, pero él volverá a ser lobo porque no pudo ni supo volverse un hombre.

Nosotros, los seres no inhumanos, no estamos constituidos solamente por reflejos condicionados; estamos estructurados por retenciones y protenciones (Husserl, 2002), es decir, por recuerdos y deseos, imaginaciones, sueños, la capacidad de transformar el mundo y de elevarse. Entre las retenciones y las protenciones, está la vida de la atención, que es una espera. Ahora bien, la espera no es un reflejo y la atención es algo que se forma; producir atención en un ser psíquico es forzosamente participar en la individuación psíquica y colectiva y, por tanto, producir con la atención psicológica atención social, es decir, vínculo social. Las retenciones y las protenciones están dispuestas en el ser no inhumano en tanto que él lucha contra el ser inhumano que hay en él. Esto tiene que ver con el deseo y con lo que Freud (1924) llamaba la sublimación. Las retenciones son los recuerdos guardados en mí que constituyen mi historia personal. Esta sirve de apuntalamiento de mis deseos, proyecciones, porvenir o, como dice Simondon (2009), a la individuación estructuralmente inacabada que me constituye en el elemento psíquico en tanto que singularidad.

Esta estructura entre retención y protención produce atención en tanto que ella se socializa, es decir, en tanto que se inscribe en circuitos que van a permitirnos heredar la lengua, el saber, la cultura, todo eso que hace que la humanidad se eleve. Esta elevación es irreversible y me obliga: estoy obligado, si soy un matemático, a estar up to date; estoy obligado, como filósofo, a leer a Simondon, no puedo hacer filosofía como en la época de Descartes. No esperamos 
de un artista que pinte como en el quattrocento. No es un asunto de progreso, sino de genealogía. Auguste Comte y Blas Pascal han descrito admirablemente esta elevación: estar sentados en las espaldas de este gigante que es la humanidad, que ha acumulado los saberes formalizando su experiencia, permite que veamos lejos.

Ahora bien, esta formación de la atención es un proceso largo en el niño. Para que un niño de hoy esté a la altura de la formación requerida se necesitan veinte años. No era así antaño. Y esta formación no está dispensada solamente por la educación nacional ni por los padres, sino por todo un conjunto, un sistema social que llamo un sistema de cuidados5 (sorge en alemán, care en inglés). Ahora bien, las industrias culturales y cognitivas tienden a destruir todo cuidado en nuestros días: una "economía de la atención" se ha desarrollado para captar la atención por todos los medios (estando dada la competencia entre todos los media), que conduce en realidad a la destrucción de los sistemas que producen atención, por ejemplo, la identificación primaria en el niño. La destrucción de la atención se observa particularmente en las investigaciones que la psiquiatría y la pediatría estadounidenses llevan a cabo sobre el attention deficit disorder, del que sufren muchos niños norteamericanos a menudo tratados con ritalina (derivada de la cocaína) o con Prozac (antidepresor). Estos niños son cada vez menos capaces de concentrarse en algo. Son destruidos por tecnologías de captación de la atención que arruinan sus capacidades de retención y de protención.

Nadie escapa a esta saturación cognitiva y afectiva, dicho de otro modo: a la desafectación y a la descognitivación, que es una "pérdida de conocimiento", una especie de epilepsia social. Y esta es la principal "tentación" mortífera de la dominación de las tecnologías de la hipermateria al servicio del psicopoder; es esta, la economía de lo hipermaterial la que es ante todo una economía de las psicotecnologías. Una tal economía es en realidad antieconómica; destruye la economía libidinal de esta hipermaterialidad, que es el espíritu del que es responsable el ser no inhumano, allí mismo donde está siempre tentado a dejarse llevar hacia la irresponsabilidad, a dejarse arrastrar a lo que perfectamente puede concretarse en ideología y en economía política del "dejar hacer, dejar pasar" (Dufour, 2007).

La hipermateria es lo que contiene ya la posibilidad de lo que se llama el espíritu; es un estado de materia transitorio, en movimiento, un proceso que está ya siempre informado y que no se lo puede simplemente analizar, ni en tanto materia ni en tanto forma. Es energía e información. Así, a nivel nanométrico ya no hay verdaderamente sentido en distinguir las industrias de la materia y las

Bernard Stiegler elabora la idea de la socioterapia en el seno de la asociación Ars Industrialis (2020). Nota del traductor. 
industrias de la información. El proceso de información pasa por la acción de la materia, por ejemplo, con las industrias farmacéuticas de los psicotrópicos. No es asunto de azar que el psicopoder se despliegue en el momento en que la industria saca todas las consecuencias posibles de lo que aparece en estas escalas en las que la oposición de la materia y de la forma ya no tiene sentido.

Vincent Bontems: De alguna manera, usted le da vuelta a la cuestión puesto que la humanidad es —-según usted—ante todo un proceso de materialización que instaura un largo circuito de formación de su psiquismo a través de los soportes materiales. Pero se ha vuelto posible, en el momento de doblar el siglo $\mathrm{XX}$, hacer nacer "cortocircuitos", es decir, actuar directamente en la temporalidad del pensamiento. Lo que designaría, en nuestros días, la tentación de lo inmaterial o, más bien, de la hipermateria sería sustituir todos los circuitos largos heredados de la historia (que implicaban siempre una cierta forma de experiencia de la resistencia de la materia) por los circuitos cortos, por lo inmediato que destruye la experiencia.

¿No se podría, sin embargo, dar otro sentido a la línea de partición entre material e inmaterial — como lo propone André Gorz (2003) — designando como "inmaterial" el dominio de la economía donde (contrariamente a lo que ha sido siempre el caso en la economía material) el criterio de la "rareza" (Sartre, 1979) ya no tuviera curso dado que acá es posible compartir un bien sin tenerlo que dividir? El término "tentación" tomaría, entonces, una connotación positiva, al menos más subversiva con respecto al sistema que usted describe porque, según Gorz (2003), es un modo de producción y de consumo socialista (donde cada quien participa en la medida de sus medios y recibe a cambio la totalidad de lo que se produce) el que parece más prolijo, y no la reproducción de los oligopolios de la economía capitalista.

Bernard Stiegler: Leí con un inmenso interés el trabajo de Gorz. La apuesta que él acepta es importante: son las implicaciones de la "reproductibilidad a costo casi nulo" (a diferencia de la reproducibilidad material clásica que siempre tenía un costo energético nada despreciable). Esto cambia la mano. Pero no hay ninguna razón para llamar a eso lo "inmaterial". Por el contrario, se necesitan muchos materiales para producir esos estados de materia, incluso si — con la ayuda de la miniaturización-se tiene la tendencia a olvidarlos. Estos aparatos son extremadamente sofisticados y cuesta caro producirlos. El problema es, pues, razonar sobre el conjunto de los factores, o al menos tratar de olvidarlos lo menos posible. La reproductibilidad a costo infinitesimal supone, en primer lugar, un aparato de reproducción. Lo que se duplica es material y lo que lo duplica también lo es. 
Sin embargo, esta economía de la reproductibilidad a costo casi nulo permite en efecto encarar otra cosa distinta de la organización capitalista actual. ¿Será, por tanto, una renovación del socialismo de la que se reclaman André Gorz (2003) o Antonio Negri (2007)? No lo pienso. No es un modelo socialista, sino un nuevo tipo de capitalismo el que hay que inventar. La división del trabajo, la inversión en la investigación y la innovación, la formación de mercados de masa, todo esto pertenece propiamente al capitalismo. El socialismo supondría la colectivización de medios de producción, y no creo que sea a esto a lo que asistamos hoy. La cooperación no es la colectivización. Yo mismo estoy por los modelos cooperativos sin por ello estar animado por un pensamiento colectivista. Por lo demás, pienso que este no es el problema actual — saber si es socialista o capitalista-, incluso si sigue siendo una cuestión para el porvenir.

El capitalismo no durará eternamente por una razón extremadamente simple: nada dura eternamente, y lo que apareció muy recientemente desaparecerá muy próximamente. Gorz (2003) dice la misma cosa, pero "muy próximamente" puede querer decir en uno o dos siglos... No estamos aún ahí. De hecho, Gorz tiene una interpretación socialista del problema, pero no tiene su teoría. No es la misma cosa. Estoy de acuerdo con él haciendo dos reservas: no se trata de lo inmaterial y no se puede hacer el anuncio de la muerte del capitalismo. Por otro lado, no hay duda de que se necesita inventar nuevos derechos y que precisamos cuestionar las limitaciones del acceso a los bienes culturales y al conocimiento. Me sitúo evidentemente a su lado en esta lucha. Y no se puede sino deplorar la ausencia total de lucidez de los responsables políticos y económicos franceses a este respecto que, por lo demás, lo único que hacen es repetir la actitud de los maestros forjadores lorenos del siglo XIX. Como ellos o, más bien, como el Estado de esa época lo pagarán caro (Stiegler, 2006, pp. 15, 21-25).

Vincent Bontems: ¿Podría usted —para terminar- desarrollar más la idea según la cual existe un lazo entre el desarrollo de los medios de almacenamiento de la memoria - lo que usted llama la "retención terciaria"- y la constitución de la psiquis humana? Freud (1924), al que usted ha evocado brevemente con respecto a la sublimación, hablaba de la memoria como de una "pizarra mágica" (wunderblock) sobre la cual se escribe y se borra cuando se levanta la primera capa, pero que conserva la inscripción en un estrato más profundo. ¿Le parece pertinente esta "metáfora de la memoria", para retomar la expresión de Douwe Draaisma (2001)?

Bernard Stiegler: Esta pregunta exigiría una respuesta mucho más profunda y corro el riesgo de frustrar al lector. Escribí sobre la resurgencia de esta metáfora 
del wunderblock en Más allá del principio del placer de Freud (1924)6 (la primera nota sobre el wunderblock es un texto precoz de antes de 1900, cuando Freud trataba de elaborar su teoría de las neuronas $\Psi$ y $\Phi)$. Mucho más tarde, después de la Primera Guerra mundial, trata de hacer una teoría de lo que él llama el sistema percepción-consciencia. En este sentido, reencuentra la idea del wunderblock y un cuestionamiento que era ya el de Platón en el Teeteto: para poder percibir es necesario que mi consciencia borre las huellas de la percepción a medida que ellas se producen al mismo tiempo que las conserva, dicho de otro modo, haciéndolas descender a la memoria. Pero, en realidad, Freud no llega a pensar la relación percepción-consciencia porque no comprendió (como no lo hicieron ni Platón ni Kant) la diferencia entre las retenciones primarias y las retenciones secundarias. Piensa que toda retención es forzosamente un recuerdo, aunque hay retenciones en el presente. Es lo que evidencia Husserl (2002) cuando retoma el análisis de Agustín de Hipona en las Confesiones.

Hay retenciones en el presente, y la percepción es precisamente una de ellas: la percepción que puede estar atenta en esto es ya una retención sin ser por ello memoria. En este momento usted me escucha y usted es más o menos consciente de lo que yo le estoy diciendo, y atento en función de las retenciones primarias que es capaz de construir. La construcción de su retención depende ella misma de sus retenciones secundarias, es decir, de lo que ya conoce o no. Pero el propio Freud se aferra a la metáfora del wunderblock o a su recuperación en Más allá del principio del placer. Por una razón que Jacques Derrida (1989) ha explicado muy bien en "Freud y la escena de la escritura"7, él no llega a pensar el wunderblock propiamente dicho; explota su metáfora pero sin apercibirse de que, sin este wunderblock, no habría eso de lo que habla y que siempre hay ya una especie de wunderblock actuando cuando hablamos de pensamiento.

Es la materialidad de lo que yo llamo la "retención terciaria". Esta es lo empírico, lo material. Y lo que se llama trascendental está del lado del que desea, es proyectado por su intencionalidad como a-tención que dispone re-tenciones y pro-tenciones sobre los objetos del deseo de quien "intencionaliza", y es así como lo imaginario (se) proyecta (en) lo real vía lo simbólico. El wunderblock y el sello de cera de Platón son metáforas porque no se tenía (todavía) teoría de las retenciones terciarias, es decir, de la materialización del tiempo espacializado $\mathrm{y}$, dicho de otro modo, de la hipermateria. $\mathrm{Y}$ es a esto a lo que se consagra lo más claro de mi tiempo. Como usted lo sugería, esta escena de la escritura del deseo, que es la materialización como retención, es también la de la sublimación en la individuación psíquica y colectiva.

$\mathrm{Al}$ respecto, véase Stiegler (2005). Nota del traductor.

Stiegler (2001) hace un comentario al respecto. Nota del traductor. 
Ahora bien, la sublimación está en nuestros días muy amenazada por las tentaciones de manipulación que las retenciones terciarias hacen posibles (como hipermateria). Estamos en el corazón de un peligrosísimo proceso de desublimación. Tomemos el ejemplo de esa escandalosa campaña de la cadena Canal J que se exhibe en los muros del metro; se ve en ella ridiculizado al padre o al abuelo, y se proclama que la cadena en cuestión debe reemplazarlos. Es la destrucción de la función simbólica del padre y del abuelo en nombre del avasallamiento del "tiempo de cerebro disponible" de los niños, que entraña tantos trastornos de la atención: es la destrucción del proceso de identificación primaria y la producción de un cerebro sin consciencia. Los niños no se construyen ya, en primer lugar, en relación con sus padres y los otros seres humanos al lado de los que están, sino enfrente de la televisión. Ahora bien, sin identificación primaria no podría haber acá libido, ni psíquica ni colectiva; nada de philia en el sentido en que Aristóteles hace de ella la condición de toda sociedad no inhumana. Un cerebro sin consciencia produce un hombre inhumano. La inhumanización de los niños no es solamente una gran y funesta tentación de nuestro tiempo; se ha vuelto lo ordinario de nuestra miseria.

\section{Referencias}

Ars Industrialis. (2020, 22 de julio). Ars Industrialis. Association internationale pour une politique industrielle des technologies de l'esprit. http://www.arsindustrialis.org/

Benjamin, W. (1982). La obra de arte en la época de la reproductibilidad técnica. En W. Benjamin, Discursos interrumpidos I. Taurus.

Bontems, V. (2008). À propos de l'hypermatériau. En B. Stiegler (comp.), L'économie de l'hypermatériel et psychopouvoir (pp. 1-46). Mille et Une Nuits.

Deleuze, G. (2003). Foucault. Paidós.

Derrida, J. (1989). Freud y la escena de la escritura. En J. Derrida (ed.), La escritura y la diferencia (pp. 271-317). Anthropos.

Draaisma, D. (2001). Metaphors of Memory: an History of Ideas about the Mind. Cambridge University Press.

Dufour, D. (2007). Le Divin Marché. La révolution culturelle libérale. Denöel.

Freud, S. (1924). El yo y el ello. Biblioteca Nueva.

Goody, J. (1977). La razón gráfica. La domesticación del pensamiento salvaje. Akal.

Gorz, A. (2003). L'Immatériel. Connaissance, valeur et capital. Éditions Galilée.

Husserl, E. (2002). Lecciones para una fenomenología de la consciencia íntima del tiempo. Editorial Trotta. Lemoine, P. (2007). La Nouvelle Origine. Nouveaux Débats publics.

Negri, A. (2007). Goodbye mister socialism. Seuil.

Sartre, J. (1979). Crítica de la razón dialéctica. Losada. 
Simondon, G. (2007). El modo de existencia de los objetos técnicos. Prometeo.

Simondon, G. (2009). La individuación a la luz de las nociones de forma y de información. La Cebra/Cactus.

Sloterdijk, p. (2000). La Domestication de l'être. Mille et une nuits.

Stiegler, B. (2001). Derrida y Tecnología: La fidelidad en los límites de la deconstrucción y la prótesis de la Fe. En T. Cohen (ed.), Jacques Derrida y las Humanidades. Cambridge University Press.

Stiegler, B. (2002). La Técnica y el tiempo 1. El pecado de Epimeteo. Hiru.

Stiegler, B. (2004a). La técnica y el tiempo III. El tiempo del cine y la cuestión del malestar. Hiru.

Stiegler, B. (2004b). De la misère symbolique 1. L'époque hyperindustrielle. Éditions Galilée.

Stiegler, B. (2005). De la misère symbolique 2. La catastrophè du sensible. Éditions Galilée.

Stiegler, B. (2006). La télécracie contre la démocratie. Lettre ouverte aux représentants politiques. Flammarion.

Stiegler, B. (2008). Prendre soin: de la jeunesse et des générations. Flammarion. 